\title{
PERFECT RECONSTRUCTION MODULATED FILTER BANKS WITH SUM OF POWERS- OF-TWO COEFFICIENTS
}

\author{
S. C. Chan, W. Liu and K. L. Ho \\ Department of Electrical and Electronic Engineering, \\ The University of Hong Kong, \\ Pokfulam Road, Hong Kong \\ email: scchan@eee.hku.hk
}

\begin{abstract}
In this paper, a new family of multiplier-less modulated filter banks, called the SOPOT MFB, is presented. The coefficients of the proposed filter banks consist of sum of powers-of-two coefficients (SOPOT), which require only simple shifts and additions for implementation. The modulation matrix and the prototype filter are derived from a fast DCT-IV algorithm of Wang [10] and the lattice structure in [1]. The design of the SOPOT MFB is performed using the genetic algorithm(GA). An 16channel SOPOT MFB with $34 \mathrm{~dB}$ stopband attenuation is given as an example, and its average number of terms per SOPOT coefficient is only 2.6 .
\end{abstract}

\section{INTRODUCTION}

Perfect Reconstruction (PR) critically decimated filter banks have important applications in speech, audio and image processing. Fig. 1(a) shows the block diagram of a critically decimated uniform $M$-channel filter banks. The theory and design of $M$-channel PR maximally decimated uniform filter bank has been extensively studied [1]. The cosine modulated filter banks (CMFB) [2] and the lapped transforms [3], [4], [5] are two efficient classes of filter banks with low implementation complexity and good performance. Recently, there is increasing interest in designing filter banks with low implementation complexity. Approaches based on the sum of power-of-two (SOPOT) coefficients [6,9] or integer coefficients [7] have been proposed. With the use of SOPOT, coefficient multiplications can be implemented with simple shifts and additions. In [7], a subspace approach was proposed to design the prototype filter of the CMFB with integer coefficients. Since integer coefficient filter banks require only integer arithmetic (additions and possibly multiplications), the implementation of the filter banks is greatly simplified. Also, if sufficient word length is used to represent the intermediate data, the round-off error can completely be eliminated.

In this paper, a new family of modulated filter bank with. sum of powers-of-two coefficients, called SOPOT-MFB, is developed. The SOPOT-MFB is based on our previous work in [8], where it was shown that modulated filter banks with perfect reconstruction can be obtained by using modulations other than the conventional cosine modulation. Unfortunately, satisfactory design of the modulations have not been obtained. In this work, the SOPOT modulation matrix and prototype filter are 0-7803-5482-6/99/\$10.00 @2000 IEEE derived from a fast DCT-IV algorithm of Wang [10] and the lattice structure in [1]. The design of the SOPOT MFB is performed using the genetic algorithm. An 16channel SOPOT MFB with $34 \mathrm{~dB}$ stopband attenuation is given as an example, and its average number of terms per SOPOT coefficient is 2.6 .

The rest of the paper is orgainized as follows : Section II is a brief overview of the theory of modulated filter banks. The construction of the SOPOT modulation matrix and the design of the SOPOT MFB will be described in Section III and IV, respectively. A design example is shown in Section V. A summary of the results is given in Section VI, the conclusion.

\section{THEORY OF MFB}

In CMFB, the analysis filters $f_{k}(n)$ and synthesis filters $g_{k}(n)$ are derived from a prototype filter $h(n)$ by cosine modulation $c_{k, n}\left(\bar{c}_{k, n}\right)$ :

$$
\begin{gathered}
f_{k}(n)=h(n) c_{k, n}, g_{k}(n)=h(n) \bar{c}_{k, n} \\
k=0,1, \ldots M-1 ; n=0,1, \ldots, N-1,
\end{gathered}
$$

where $M$ is the number of channels and $N=2 m M$ is the length of the filter. Two different modulations can be used and without loss of generality, here we will consider the modulation proposed in [2] :

$$
\begin{aligned}
& c_{k, n}=2 \cos \left((2 k+1) \frac{\pi}{2 M}\left(n-\frac{N-1}{2}\right)+(-1)^{k} \frac{\pi}{4}\right) \\
& \bar{c}_{k, n}=2 \cos \left((2 k+1) \frac{\pi}{2 M}\left(n-\frac{N-1}{2}\right)-(-1)^{k} \frac{\pi}{4}\right)
\end{aligned}
$$

Let $H(z)=\sum_{q=0}^{2 M-1} z^{-q} H_{q}\left(z^{2 M}\right)$ be the type-I polyphase decomposition of the prototype filter and $F_{k}(z)$ the $z$ transform of $f_{k}(n)$. It can be shown that

$$
F_{k}(z)=\sum_{q=0}^{2 M-1} c_{k, q} z^{-q} H_{q}\left(-z^{2 M}\right) .
$$

The analysis filter bank can be expressed in matrix form

$$
\begin{aligned}
& \text { as: } f(z)=\hat{C}_{A}\left[\begin{array}{c}
h_{0}\left(z^{2 M}\right) \\
z^{-M} h_{1}\left(z^{2 M}\right)
\end{array}\right] \boldsymbol{e}_{M}(z)=\boldsymbol{E}\left(z^{M}\right) \boldsymbol{e}_{M}(z),(4) \\
& \text { where } \boldsymbol{e}_{M}^{T}(z)=\left[\begin{array}{llll}
1 & z^{-1} & \ldots & z^{-(M-1)}
\end{array}\right] \text {, } \\
& f(z)=\left[F_{0}(z), F_{1}(z), \ldots, F_{M-1}(z)\right]^{T}, \\
& \boldsymbol{h}_{0}(z)=\operatorname{diag}\left[H_{0}(-z), H_{1}(-z), \ldots, H_{M-1}(-z)\right] \\
& h_{1}(z)=\operatorname{diag}\left[H_{M}(-z), H_{M+1}(-z), \ldots, H_{2 M-1}(-z)\right]
\end{aligned}
$$


$\boldsymbol{E}(z)$ is the polyphase matrix and $\hat{\boldsymbol{C}}_{\boldsymbol{A}}$ is a $(M \times 2 M)$ modulation matrix given by $\left[\hat{C}_{A}\right]_{k, n}=c_{k, n} . \hat{C}_{A}$ can be further partitioned as $\hat{C}_{\boldsymbol{A}}=(-1)^{q}\left[\begin{array}{ll}A_{0} & \boldsymbol{A}_{1}\end{array}\right](q=m / 2$ for $m$ even and $q=(m-1) / 2$ for $m$ odd $)$ [2]:

$$
\begin{aligned}
& A_{0}=\sqrt{M} C_{M}^{I V}\left(\boldsymbol{I}_{M}+(-1)^{m-1} J_{M}\right), \\
& A_{1}=(-1)^{m-1} \sqrt{M} C_{M}^{I V}\left(I_{M}-(-1)^{m-1} J_{M}\right),
\end{aligned}
$$

where $C_{M}^{N}(k, n)=\sqrt{2 / M} \cos ((k+1 / 2)(n+1 / 2) \pi / 2 M)$ is the type-IV DCT. It can be shown that the system is PR if the prototype filter satisfies

$$
H_{k}(z) H_{2 M-k-1}(z)+H_{M+k}(z) H_{M-k-1}(z)=c z^{-s_{k}}
$$

It has been shown in [8] that the PR condition of the modulated filter bank is still given by (6) if

$$
\hat{C}_{A}^{T} \hat{C}_{A}=2 M\left[\begin{array}{cc}
I_{M} \pm J_{M} & 0 \\
0 & I_{M} \mp J_{M}
\end{array}\right],
$$

where $I_{M}$ and $J_{M}$ are $(M \times M)$ identity and exchange matrices, respectively. The result in [8] was originally derived for orthogonal MFB but it is still valid for the the more general biorthogonal PR condition (6). The general solutions for $A_{0}$ and $A_{1}$ are (up to a scaling)

$$
\begin{aligned}
A_{0} & =\sqrt{M} U_{M}\left(I_{M} \pm J_{M}\right), \\
\text { and } A_{1} & = \pm \sqrt{M} U_{M}\left(I_{M} \mp J_{M}\right),
\end{aligned}
$$

for some unitary matrix $U_{M}$. If the modulation is not orthogonal, (6) will still be valid if the analysis modulation matrix $\hat{C}_{A}$ and synthesis modulation matrix satisfy $\hat{C}_{s}$ satisfy

$$
\hat{C}_{S}^{T} \hat{C}_{A}=2 M J_{2 M}\left[\begin{array}{cc}
I_{M} \pm J_{M} & 0 \\
0 & I_{M} \mp J_{M}
\end{array}\right],
$$

where $\hat{C}_{s}$ is the modulation matrix of the type-I polyphase decomposition of the synthesis filters:

$$
\boldsymbol{g}(z)=\left[G_{0}(z), \ldots G_{M-1}(z)\right]^{T}=\hat{C}_{S}\left[\begin{array}{c}
h_{0}\left(z^{2 M}\right) \\
z^{-M} h_{1}\left(z^{2 M}\right)
\end{array}\right] e_{M}(z) .
$$

It can be shown that $\hat{C}_{A}$ and $\hat{C}_{S}$ are given by (up to a scaling)

$$
\begin{gathered}
C_{A}=\sqrt{M} U_{M}\left[I_{M} \pm J_{M}, \pm\left(I_{M} \mp J_{M}\right)\right], \\
C_{S}=\sqrt{M}\left(U_{M}^{-1}\right)^{T}\left[I_{M} \pm J_{M}, \pm\left(I_{M} \mp J_{M}\right)\right] J_{2 M},
\end{gathered}
$$

where $U_{M}$ is now an arbitrary nonsingular matrix. Without loss of generality, we will consider the case where

$$
\begin{aligned}
& C_{A}=\sqrt{M} U_{M}\left[I_{M}+J_{M},\left(I_{M}-J_{M}\right)\right], \\
& C_{S}=\sqrt{M}\left(U_{M}^{-1}\right)^{T}\left[I_{M}+J_{M},\left(I_{M}-J_{M}\right)\right] J_{2 M} .
\end{aligned}
$$

\section{SOPOT MODULATION}

To derive the SOPOT modulation, the simplest way is to quantize the coefficients in the type-IV DCT. The inverse of the quantized matrix, however, cannot in general be expressed in terms of SOPOT coefficients. Let's consider the simple example:

$$
\boldsymbol{R}_{\theta}=\left[\begin{array}{cc}
\cos \theta & \sin \theta \\
\sin \theta & -\cos \theta
\end{array}\right] .
$$

If $\cos \theta$ and $\sin \theta$ are expressed directly in terms of SOPOT coefficients, say $\alpha$ and $\beta$. The inverse of $\tilde{\boldsymbol{R}}_{\theta}=\left[\begin{array}{cc}\alpha & \beta \\ \beta & -\alpha\end{array}\right]$ is $\tilde{\boldsymbol{R}}_{\theta}^{-1}=\frac{1}{\alpha^{2}+\beta^{2}}\left[\begin{array}{cc}\alpha & \beta \\ \beta & -\alpha\end{array}\right]$. As $\alpha$ and $\beta$ are SOPOT coefficients, the term $\left(\alpha^{2}+\beta^{2}\right)^{-1}$ cannot in general be expressed as a SOPOT coefficient. The basic idea of the proposed multiplier-less modulation is based on the following factorization of $\boldsymbol{R}_{\theta}$ and its inverse

$$
\left.\left.\boldsymbol{R}_{\theta}=\boldsymbol{R}_{\theta}^{-1}=\left[\begin{array}{cc}
1 & -\tan (\theta / 2) \\
0 & 1
\end{array}\right] \begin{array}{cc}
1 & 0 \\
\sin \theta & 1
\end{array}\right] \begin{array}{cc}
1 & \tan (\theta / 2) \\
0 & -1
\end{array}\right] \text {. }
$$

Since the factorizations of $\boldsymbol{R}_{\theta}$ and $\boldsymbol{R}_{\theta}^{-1}$ involve the same set of coefficients, i.e. $\tan (\theta / 2)$ and $\sin \theta$, they can be directly quantized to SOPOT coefficients. To get the SOPOT modulation, we start with the fast algorithm of Wang [10], which factorizes the DCT-IV into a product of $2 \ell+1$ sparse matrices as follows:

$$
C_{M}^{N}=Q_{M} V_{M}(\ell)\left[\prod_{i=1}^{\ell-1} K_{M}(\ell-i) V_{M}(\ell-i)\right] \boldsymbol{H}_{M}, \quad \ell=\log _{2} M,
$$

where

$$
\begin{aligned}
& \left.\boldsymbol{H}_{M}=\boldsymbol{P}_{M}\left(\boldsymbol{P}_{M / 2} \oplus \overline{\boldsymbol{P}}_{M / 2}\right) \cdot \operatorname{diag}\left(\boldsymbol{P}_{M / 4}, \overline{\boldsymbol{P}}_{M / 4}, \boldsymbol{P}_{M / 4}, \overline{\boldsymbol{P}}_{M / 4}\right)_{4}\right) \\
& \cdots \operatorname{diag}\left(\boldsymbol{P}_{4}, \overline{\boldsymbol{P}}_{4}, \cdots, \boldsymbol{P}_{4}, \overline{\boldsymbol{P}}_{4}\right), N \geq 4 \text {, }
\end{aligned}
$$

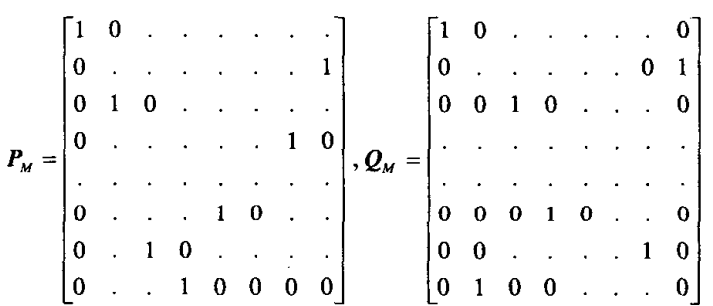

$\overline{\boldsymbol{P}}_{M}$ is obtained by reversing both the rows and columns of $\boldsymbol{P}_{M}, Q_{M}$ is a permutation matrix that changes the odd-numbered components of the vector into a reversed order. $\boldsymbol{K}_{M}(i), \quad i=1,2, \ldots, \ell-1$, are block diagonal matrices given by

$$
\boldsymbol{K}_{M}(i)=(1 / \sqrt{2}) \cdot \operatorname{diag}(\boldsymbol{B}(i), \boldsymbol{B}(i), \cdots, \boldsymbol{B}(i), \boldsymbol{B}(i)),
$$

where $B(i)=\left[\begin{array}{cc}\boldsymbol{I}_{2^{i}} & \boldsymbol{I}_{2^{i}} \\ \boldsymbol{I}_{2^{i}} & -\boldsymbol{I}_{2^{i}}\end{array}\right] \cdot \boldsymbol{V}_{M}(\ell)$ is given by

$$
\boldsymbol{V}_{M}(\ell)=\operatorname{diag}\left(\boldsymbol{T}_{1 / 4 M}, \boldsymbol{T}_{5 / 4 M}, \cdots, \boldsymbol{T}_{(2 M-3) / 4 M}\right),
$$

where $\quad T_{r}=\left[\begin{array}{cc}\cos r \pi & \sin r \pi \\ \sin r \pi & -\cos r \pi\end{array}\right]$. The other matrices $V_{M}(i), i=1,2, \ldots, \ell-1$, are obtained by alternating the submatrices $\boldsymbol{I}_{2^{i}}$ and $\boldsymbol{E}(i)$ in the main diagonal as follows

$$
V_{M}(i)=\operatorname{diag}\left(\boldsymbol{I}_{2^{i}}, E(i), I_{2^{i}}, \cdots, E(i)\right)
$$

where $\boldsymbol{E}(i)=\operatorname{diag}\left(\boldsymbol{T}_{1 / 2^{i+1}}, \boldsymbol{T}_{5 / 2^{i+1}}, \cdots, \boldsymbol{T}_{\left(2^{i+1}-3\right) / 2^{i+1}}\right)$. 
Replacing $T_{r}$ by $\left.\left.S_{r}=\left[\begin{array}{cc}1 & -\beta_{r} \\ 0 & 1\end{array}\right] \begin{array}{cc}1 & 0 \\ \alpha_{r} & 1\end{array}\right] \begin{array}{cc}1 & \beta_{r} \\ 0 & -1\end{array}\right]$, with $\alpha_{r}$ and $\beta_{r}$ SOPOT coefficients, the desired SOPOT modulation matrix is obtained. Let $\boldsymbol{R}_{M}(i)$ be the matrix obtained by replacing $T_{r}$ in $V_{M}(i)$ by $S_{r}$. Because $\boldsymbol{S}_{r}^{-1}=\boldsymbol{S}_{\boldsymbol{r}}, \quad \boldsymbol{R}_{M}^{-1}(i)=\boldsymbol{R}_{M}(i)$. The SOPOT modulation matrix $U_{M}$ and its inverse are given by:

$$
\begin{gathered}
U_{M}=Q_{M} \boldsymbol{R}_{M}(\ell)\left[\prod_{i=1}^{\ell-1} K_{M}(\ell-i) \boldsymbol{R}_{M}(\ell-i)\right] \boldsymbol{H}_{M} \\
\text { and } \boldsymbol{U}_{M}^{-1}=\boldsymbol{H}_{M}^{T}\left[\prod_{i=1}^{\ell-1} \boldsymbol{R}_{M}(i) K_{M}(i)\right] \boldsymbol{R}_{M}(\ell) Q_{M}
\end{gathered}
$$

\section{DESIGN OF THE MFB}

To design the prototype filter with SOPOT coefficients, we use the lossless lattice structure proposed in [1] (Figure 2). The lattice coefficients are represented as:

$$
\begin{aligned}
& \alpha_{q, n}=\sum_{k=1}^{p_{n}} a_{k} 2^{b_{k}} \\
& a_{k} \in\{-1,1\} b_{k} \in\{l, \ldots, 1,0,-1, \ldots,-l\} .
\end{aligned}
$$

$l$ is a positive integer, which determines the range of the coefficient, $p_{n}$ is the number of terms used in each coefficient. The minimax objective function is used in the design of the prototype filter:

$$
D_{h}=\max \left\|H\left(e^{j \omega}\right)|-| H_{d}\left(e^{j \omega}\right)\right\|,
$$

where $H(z)$ and $H_{d}(z)$ are respectively the actual and ideal frequency responses of the prototype filter. The modulation matrix is designed using a similar minimax objective function

$$
D_{M}=\max _{0 \leq k \leq M-1}\left\{E_{k}=\max \left\|F_{k}\left(e^{j \omega}\right)|-| \hat{F}_{k}\left(e^{j \omega}\right)\right\|\right\},
$$

where $F_{k}\left(e^{j \omega}\right)$ and $\hat{F}_{k}\left(e^{j \omega}\right)$ are respectively the actual and ideal frequency responses of the $k$-th analysis filter of the SOPOT MFB.The SOPOT coefficients are found by minizing $D_{h}$ and $D_{M}$ using the Genetic Algorithm.

\section{DESIGN EXAMPLES}

Fig. 3(a) and 3(b) show, respectively, the frequency responses of the prototype and the analysis filters of an 16-channel SOPOT MFB. The cutoff frequency of the prototype filter is $\omega_{s}=0.075 \pi$ and its stopband attenuation is $37 \mathrm{~dB}$. Because of the linear-phase property of the prototype filter, there are altogether eight different lattices. Polyphase components $H_{q}(z)$ and $H_{q+16}(z)$ are derived from the $q$-th lattice, and all of them have two lattice coefficients $\alpha_{q, 1}, \alpha_{q, 2}$, i.e. $(m=2)$.

\begin{tabular}{|c|c|c|}
\hline$q$ & $\alpha_{q, 1}$, & $\alpha_{q, 2}$ \\
\hline 0 & $2^{2}-2^{-1}$ & $-2^{2}+2^{-4}$ \\
\hline 1 & $2^{1}+2^{-1}+2^{-5}$ & $-2^{2}-2^{0}-2^{-2}$ \\
\hline 2 & $2^{1}+2^{-1}$ & $-2^{2}-2^{0}-2^{-1}-2^{-3}$ \\
\hline 3 & $2^{1}$ & $-2^{3}-2^{0}$ \\
\hline 4 & $2^{0}+2^{-1}+2^{-4}$ & $-2^{4}-2^{2}$ \\
\hline
\end{tabular}

\begin{tabular}{|l|c|c|}
\hline 5 & $2^{0}+2^{-1}-2^{-6}$ & $-2^{5}+2^{1}$ \\
\hline 6 & $2^{0}+2^{-2}$ & $-2^{4}-2^{1}$ \\
\hline 7 & $2^{0}+2^{-4}$ & $-2^{5}-2^{4}$ \\
\hline
\end{tabular}

The SOPOT modulation matrix, $U_{16}$, is given by

$$
\begin{gathered}
U_{16}=Q_{16} R_{16}(4) K_{16}(3) R_{16}(3) K_{16}(2) R_{16}(2) \\
K_{16}(1) R_{16}(1) H_{16},
\end{gathered}
$$

where $R_{16}(4)=\operatorname{diag}\left\{S_{1 / 64}, S_{5 / 64}, S_{9 / 64}, S_{13 / 64}\right.$,

$$
\begin{array}{r}
\left.S_{17 / 64}, S_{21 / 64}, S_{25 / 64}, S_{29 / 64}\right\}, \\
R_{16}(3)=\operatorname{diag}\left\{I_{8}, S_{1 / 16}, S_{5 / 16}, S_{9 / 16}, S_{13 / 16}\right\} \\
R_{16}(2)=\operatorname{diag}\left\{I_{4}, S_{1 / 8}, S_{5 / 8}, I_{4}, S_{1 / 8}, S_{5 / 8}\right\} \\
R_{16}(1)=\operatorname{diag}\left\{I_{2}, S_{1 / 4}, I_{2}, S_{1 / 4}, I_{2}, S_{1 / 4}, I_{2}, S_{1 / 4}\right\}
\end{array}
$$

As mentioned earlier, each $S_{r}$ is characterized by two parameters $\alpha_{r} \quad$ and $\beta_{r}$. Noting that $T_{x+1 / 2}=T_{x} \cdot\left[\begin{array}{cc}0 & 1 \\ -1 & 0\end{array}\right], \quad$ then $\quad S_{x+1 / 2}=S_{x} \cdot\left[\begin{array}{cc}0 & 1 \\ -1 & 0\end{array}\right]$, $(\mathrm{x}=1 / 16,5 / 16,1 / 8), \quad S_{9 / 16}, S_{13 / 16}$, and $S_{5 / 16}$ can be derived from $S_{1 / 16} S_{5 / 16}$, and $S_{1 / 8}$, respectively. The number of variables is therefore reduced and their details are as follows:

\begin{tabular}{|c|c|c|}
\hline$r\left(S_{r}\right)$ & $\alpha_{r}$ & $\beta_{r}$ \\
\hline $1 / 64$ & $2^{-4}$ & $2^{-5}$ \\
\hline $5 / 64$ & $2^{-2}-2^{-6}-2^{-7}$ & $2^{-3}-2^{-7}$ \\
\hline $9 / 64$ & $2^{-1}-2^{-4}$ & $2^{-2}-2^{-6}-2^{-7}$ \\
\hline $13 / 64$ & $2^{-1}+2^{-4}+2^{-6}+2^{-7}$ & $2^{-2}+2^{-4}+2^{-6}$ \\
\hline $17 / 64$ & $2^{-1}+2^{-2}+2^{-6}+2^{-7}$ & $2^{-1}-2^{-4}+2^{-7}$ \\
\hline $21 / 64$ & $2^{0}-2^{-3}-2^{-7}$ & $2^{-1}+2^{-4}-2^{-7}$ \\
\hline $25 / 64$ & $2^{0}-2^{-4}$ & $2^{-1}+2^{-2}-2^{-5}$ \\
\hline $29 / 64$ & $2^{0}-2^{-7}$ & $2^{0}-2^{-3}-2^{-6}$ \\
\hline $1 / 16$ & $2^{-3}+2^{-4}+2^{-6}+2^{-7}$ & $2^{-3}-2^{-6}$ \\
\hline $5 / 16$ & $2^{-1}+2^{-2}+2^{-4}$ & $2^{-1}+2^{-5}$ \\
\hline $1 / 8$ & $2^{-2}+2^{-3}$ & $2^{-3}+2^{-4}+2^{-7}+2^{-8}$ \\
\hline $1 / 4$ & $2^{-1}+2^{-2}-2^{-4}+2^{-6}$ & $2^{-1}-2^{-4}-2^{-6}-2^{-7}$ \\
\hline
\end{tabular}

The average number of terms per SOPOT coefficient is 2.6 .

\section{CONCLUSION}

In this paper, a new family of multiplier-less modulated filter banks, called the SOPOT MFB, is presented. The coefficients of the proposed filter banks consist of sum of powers-of-two coefficients (SOPOT), which require only simple shifts and additions for their implementation. The modulation matrix and the prototype filter are derived from a fast DCT-IV algorithm of Wang [10] and the lattice structure in [1]. The genetic algorithm is used to search for the SOPOT coefficients of the filter banks. An 16-channel SOPOT MFB with $34 \mathrm{~dB}$ stopband attenuation is given as an example, and its average number of terms per SOPOT coefficient is only 2.6 .

\section{REFERENCES}

[1] P.P. Vaidyanathan, Multirate systems and Filter banks, Engltwood Cliffs: Printice-Hall, 1993. 
[2] R. D. Koilpillai and P. P. Vaidyanathan, "CosineModulated FIR filter banks satisfying perfect reconstruction," IEEE Trans. ASSP-40, April 1992, pp. 770-783.

[3] H. S. Malvar, "Extended Lapped Transforms: Properties, Applications, and Fast Algorithms," IEEE Trans. SP, Vol.40, Nov. 1992, pp. 2703-2714.

[4] H. S. Malvar, "Lapped transforms for efficient transform/subband coding," IEEE Trans. ASSP-38, June 1990, pp. 969-978.

[5] R. L. de Queiroz, and T. Q. Nguyen "The GenLOT: Generalized Linear Phase lapped orthogonal Transform", IEEE Trans. SP, Vol. 44, March 1996, pp. 497-507.

[6] S. Sriranganathan, D. R. Bull, D. W. Redmill, "The design of Low Complexity Two-Channel LatticeStructure Perfect-Reconstruction Filter Banks Using Genetic Algorithm". Proc. of ISCAS '97., vol.4, pp.23932396, June 1997.

[7] A. Mertins, "Discrete-coefficients Linear-phase Prototypes For PR Cosine-Modulated Filter Banks",Proc of ICASSP' 98, Vol.3, pp.1433-1436, May 1998.

[8] S. C. Chan and C. W. Kok, "Perfect Reconstruction Modulated Filter Banks without Cosine Constraints," Proc. 1993 IEEE Int'l Conf. Acoustic Speech Signal Processing, pp. III. 189-192, April 27-29, Minneapolis, Minnesota, USA.

[9] B. R. Horng, H. Samueli and A. N. Willson, "The design of Two-Channel Lattice-Structure PerfectReconstruction Filter banks Using Powers-of-Two Coefficients"' IEEE Trans. CAS, Vol. 40, No 7, July 1993.

[10] Z. Wang, "Fast algorithms for the discrete W transform and for the discrete Fourier transform", IEEE Trans. ASSP-32, August 1984, pp. 803-816.

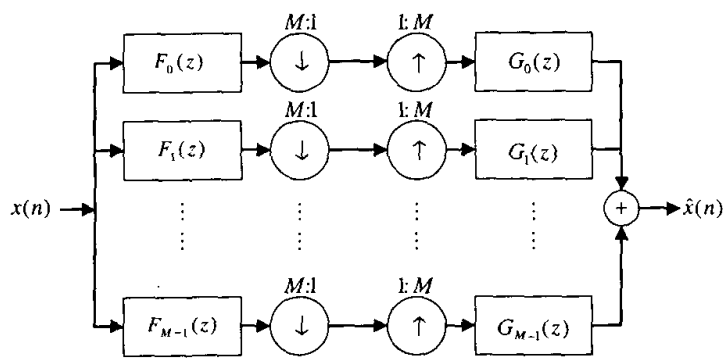

(a)

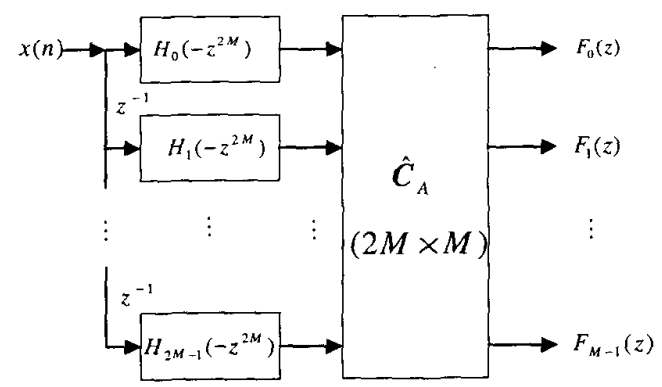

(b)
Fig. 1. Block diagrams of (a) $M$-channel uniform filter banks, (b) polyphase implementation of the cosine-modulated analysis filter bank.

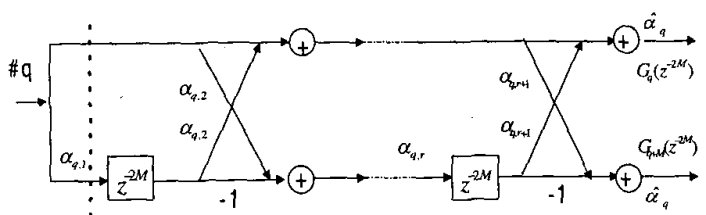

Fig. 2. Lossless lattice structure of the prototype filter in [1]

$$
\left(\hat{\alpha}_{q}=\prod_{i=1}^{r+1}\left(1+\alpha_{q, i}^{2}\right)^{-0.5}\right) \text {. }
$$

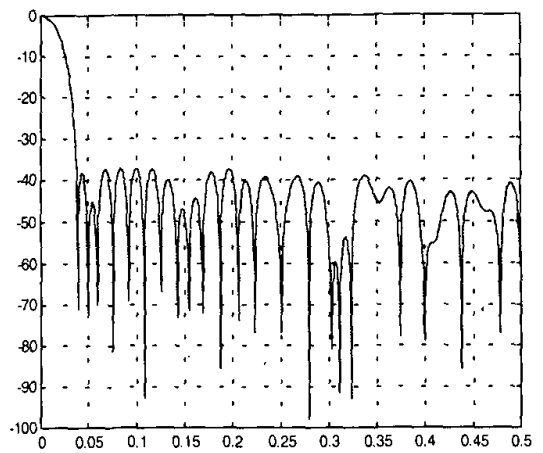

(a)

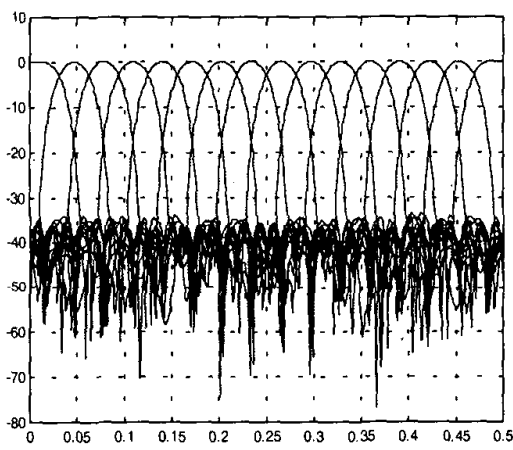

(b)

Fig. 3. Frequency responses of the 16-channel SOPOT MFB: (a) prototype filter (length $=64, \omega_{s}=0.075 \pi, A_{s}=37 d B$ ), (b) analysis filters, $A_{s}=34 d B$. 